\title{
FAIR DATA AUSTRIA - ALIGNING THE IMPLEMENTATION OF FAIR TOOLS AND SERVICES
}

\section{by Susanne Blumesberger, Raman Ganguly, Nikos Gänsdorfer, Eva Gergely,}

Alexander Gruber, Ilire Hasani-Mavriqi, Tereza Kalová, Christoph Ladurner, Therese Macher, Tomasz Miksa, Barbara Sanchéz Solís, Hermann Schranzhofer, Christiane Stork, Sarah Stryeck, Heike Töricht*

Abstract: This article gives an overview of the FAIR Data Austria project objectives and current results. In collaboration with our project partners, we work on the development and establishment of tools for managing the lifecycle of research data, including machine-actionable Data Management Plans (maDMPs), repositories for longterm archiving of research results, RDM training and support services, models, and profiles for Data Stewards and FAIR Office Austria.

Keywords: FAIR principles; next-generation repositories; reproducible research results; research data management (RDM); machine-actionability; data management plans; data stewards; RDM support and training; researcher engagement

\section{FAIR DATA AUSTRIA - ABSTIMMUNG DER IMPLEMENTIERUNG VON FAIR TOOLS UND SERVICES}

Zusammenfassung: Dieser Artikel gibt einen Überblick über die Ziele und aktuellen Ergebnisse des FAIR Data Austria-Projekts. In Zusammenarbeit mit unseren Projektpartnern arbeiten wir an der Entwicklung und Etablierung von Tools für das Management des Lebenszyklus von Forschungsdaten, einschließlich maschinenverarbeitbarer Datenmanagementpläne (maDMPs), Repositories für die Langzeitarchivierung von Forschungsergebnissen, RDM-Schulungen und -Support-Services, Modelle und Profile für Data Stewards und das FAIR Office Austria.

Schlagworte: FAIR-Prinzipien; Next-Generation Repositorien; reproduzierbare Forschungsergebnisse; Forschungsdatenmanagement (FDM); Machine-Actionability; Datenmanagementpläne (DMPs); Data Stewards; RDM-Support und -Training; Researcher Engagement

DOI: https://doi.org/10.31263/voebm.v74i2.6379

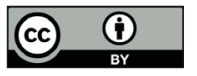

This work - excl. individual logos and illustrations - is licensed under a Creative Commons Attribution 4.0 International license 


\section{Introduction}

The FAIR Data Austria ${ }^{1}$ project is led by the Graz University of Technology (TU Graz), with TU Wien and the University of Vienna as core partners. Further cooperation partners are the Academy of Fine Arts Vienna, the Medical University of Graz, and the University of Innsbruck.

The project contributes to the strengthening of knowledge transfer between universities, industry and society and supports the sustainable implementation of the European Open Science Cloud (EOSC). In this context, the implementation of the FAIR principles ("Findable, Accessible, Interoperable and Reusable") plays a significant role. Compliance with them is ensured through (1) integrated research data management (RDM) tailored to generic and discipline-specific needs of researchers, (2) development of Next Generation repositories for research data, code, and databases, and (3) development of training and support services for efficient RDM.

For a successful RDM that complies with the FAIR principles, it is essential to support the entire lifecycle of research data - from generation to archiving - with specific expertise and a set of associated tools and services. This cannot be done as an isolated effort. The FAIR Data Austria project promotes collaboration between Austrian universities in the development of coherent and solid services for research data. This collective effort enables Austrian universities to maximize synergies and potential for resource-optimized collaboration to promote the objectives of Open Science, Open Data, Open Access, and Open Innovation and secure their role in the international research landscape.

The following chapters introduce the FAIR Data Austria tools and services and outline the current results, lessons learned and next steps.

\section{Managing the lifecycle of research data - machine-actionable Data Management Plans (maDMPs)}

Data Management Plans (DMPs) describe the data used and produced in scientific experiments by specifying where they will be stored, how to access it, and under what licenses they can be reused (Miksa et al., 2019). Machine-actionable DMPs (maDMPs) are an official recommendation of the Research Data Alliance (RDA), who defined a common way for exchange of information typically covered by DMPs in a way that can be acted upon by machines (Miksa et al., 2020). Thus, maDMPs have the potential to better integrate the stakeholders involved in RDM by facili- 
tating the exchange of information, while remaining compatible with existing funder requirements.

FAIR Data Austria develops the DAMAP ${ }^{2}$ tool, an open-source software facilitating the creation of DMPs. Machine-actionability is one of the key principles driving the development of DAMAP. To enable this, DAMAP stores information in a machine-actionable way from the beginning of data collection and is designed in a way to integrate well with other services operated by institutions that can provide or reuse information from maDMPs. For example, every DAMAP instance retrieves information on available repositories from re 3 data $^{3}$. Integration with specific services is also possible. For example, TU Wien integrates DAMAP with its personnel and project database, and provides a custom list of storage locations that offer services to its researchers. To create a DMP, DAMAP follows a questionnaire based on the Science Europe template and takes into account the requirements of the Evaluation Rubric ${ }^{4}$. Currently, DAMAP exports prefilled DMPs as MS Word documents that can be further customised and edited by researchers, who in this way do not have to face the "blank page syndrome" anymore.

During the development of DAMAP we have learned three major lessons. First, achieving the balance between user experience - how easy it is to write/generate a DMP - and the number of questions and details potentially needed is not a trivial task. It would be easy to just present the Science Europe template to researchers, but this could lead to confusion, because not all questions and topics are equally relevant for all researchers in all situations. Furthermore, the evaluation rubric requires specific information, like frequency of backups for storage used, which is not what researchers may or even should know - such information should be provided automatically depending on the storage location they choose.

This leads to the second challenge we had to face: to prefill some information automatically, we had to collect this information first-hand, e.g. on the frequency of backups of storage services provided by the universities. This resulted in discussions with various departments that required clarification of internal workflows and documents before the information was added to DAMAP. Such discussions should be planned ahead by any institution that wants to customise DAMAP.

Third, to integrate DAMAP with existing systems, common interfaces and controlled vocabularies are needed. Using CERIF ${ }^{5}$ for CRIS systems is one of the options. However, there is still no standardisation among universities. Hence, there is a need for custom integration between DAMAP and existing services, e.g. project and personnel databases running at each 
university. This can be solved by software developers, but needs to be planned, and can also require extra changes in the systems to which DAMAP connects to.

Ongoing and future work will focus in the first phase on testing DAMAP with a selected group of researchers and funders to further improve the guidance and identify further integration possibilities that reduce the need for manual input. In the second phase, we plan to deploy DAMAP at different universities and strengthen (inter-)national collaborations. In the long term, we plan to open DAMAP to everyone who is interested, and to establish a group of users who maintain the software and work jointly to improve and extend it further.

\section{Establishing repositories for research data}

Repository platforms play a critical role in the research ecosystem to preserve and share research, enable reproducibility, and enable reuse of datasets and other research output. ${ }^{6}$

TU Graz, TU Wien, and the University of Innsbruck are establishing next generation repositories for research data based on the InvenioRDM ${ }^{7}$ open-source software solution. InvenioRDM is developed as part of a large, multi-organization collaboration in partnership with CERN ${ }^{5}$, with TU Graz and TU Wien in the core-team. InvenioRDM is a turn-key RDM repository platform based on Invenio Framework and Zenodo ${ }^{6}$. It provides a wide range of features that ensure good research data practice and the implementation of the FAIR principles ${ }^{8}$.

The InvenioRDM project released the first Long-Term Support (LTS) version, also known as v6.0, in August 2021, thereby making it suitable for production systems. Supported features include creating records (any resource type and file/format size), DOI registration via DataCite, strong support for persistent identifiers (authors, affiliations, licenses, related papers/datasets), faceted search, login via institutional account, customization, styling and theming, internationalisation, restricted records, share by link, and embargo support.

A first production version of the TU Graz Repository ${ }^{9}$ was made available to all researchers at TU Graz in April 2021. With the Repository, researchers have a secure digital open-source software solution for the publication and long-term archiving of research data, publications and educational resources that can be accessed via the usual TU Graz Single Sign-on (SSO). This is the first rollout of InvenioRDM for the collaborative project 
around CERN and has been used as "sounding board" for further development and migration processes. The TU Graz Repository offers many advantages: secure data storage to prevent data loss, allocation of a DOI (Digital Object Identifier) to increase citability, efficient updating of data sets by means of a versioning system, metadata for improved findability and reuse of data, management of access rights (open, restricted, or closed access) and granting licences to regulate any future use of the data, with new features to be added on a continuous basis.

The Repository is operated by the TU Graz Library ${ }^{10}$ together with the RDM Team ${ }^{11}$. Users are provided with a detailed documentation on the deployment of the software solution, a handbook, terms of use and data protection policy.

TU Wien offers its institutional repository, TU Wien Research Data ${ }^{12}$, operated by the TU Wien Center for Research Data Management, to a closed group of selected users. By allowing only a closed group of researchers, the Center can provide individual help to researchers who are learning to use the new system, while at the same time collecting detailed feedback that is then used to prioritise developments. The first ${ }^{13}$ DOI was minted in December 2020 for the 3 TB dataset of Sentinel-1 satellite data. Since then, the data repository of TU Wien has been extended with new features on a rolling basis and improved the user interface and has been subject to security audits. The development and support team has also created exhaustive system documentation, policy documents, Service Level Agreements, etc. that are crucial for securing the repository's long-term mission. The repository will be made available for all TU Wien researchers to selfpublish their data in 2022.

The in-house repositories at TU Graz and TU Wien have been so far very well received by the researchers. The feedback collected suggests that researchers are happy to move their data into an institutional repository. An explanation of this is that although many researchers already keep their data on servers operated by the universities, the data is generally not compliant with the FAIR principles, and the responsibility to ensure this is left to them. Hence, they welcome any service that can take care of their data and makes sure it stays within the university and is not handed over to third parties, e.g. publishers.

The key difference in establishing a data repository compared to other software engineering projects is trust: Researchers must trust that their data will not be lost, e.g. if a project gets discontinued. This requires that all data received from researchers be handled with care, even during the pre-production stages in the development of the repository. 
By actively contributing to the InvenioRDM project, our developers got valuable insights into the software they operate and became key experts in the advance of the repository software. TU Graz and TU Wien can add features to their systems that are developed faster and tested better than if developed in isolation. One of the next features to be added is the "community feature" that enables grouping of records to a specific topic and collective assignment of access rights. In the case of the TU Graz Repository, the next steps are the integration of various systems (PURE, ALMA) and the development of a new data model for Open Educational Resources (OER).

\section{Implementing repositories for databases}

A repository is designed to preserve data stored in files. However, when it comes to databases, standard repositories have their limits. The new kind of repository we propose is designed to store data from databases and make data citable according to the recommendation of the RDA Data Citation working group (Rauber, 2015). This database repository will focus on relational databases, as they are widely used as a core source for data in many research disciplines. The main challenge here is not only to preserve the data itself but to also provide the common functionality of databases - including the insertion, update, and deletion of records. Users can design the database directly in the repository; in this way, the repository can be used from the beginning of the project and not only post factum. To facilitate this, a rich API (Application Programming Interface) is necessary. We are aware that such a versatile software cannot be finished during the life span of the project. For this very reason, we focus on the goal to implement a running open-source prototype.

The software's core concept and architecture is ready and will be presented at the iPRES 2021 - 17 $7^{\text {th }}$ International Conference on Digital Preservation. To store the databases, Docker containers are used, with all additional information in a separate metadata store. In the current the prototype, users can create a new database and import data from CSV files over a user interface. A Docker container is created for each new database, and changes are tracked through timestamping. Thus, for each insert, update or delete operation the record is assigned a timestamp. This way, it can be ensured that queries deliver the exact same result upon reexecution at any time in the future. Deleted records are marked as deleted. The concept follows the FAIR data principles and the RDA data citation recommendation mentioned above. 
As is well-known from similar software development projects, it is essential to keep detailed documentation of the evolution of the repositories. This has become even more evident due to several changes in the staff. The formation and changes in the team prompted the need to define the project's scope as precisely as possible, thus ensuring that a running prototype with data citation functionality is delivered by the end of the project, for without this feature the repository would amount to just another Docker infrastructure for databases. To improve our productivity, we have introduced agile software development and adopted three-week sprints as working method. Additionally, after a long period of home office due to COVID19 restrictions we have now managed to hold in-person meetings, which have been essential to solve communication issues that appeared during the time where only online meetings were possible.

Since the core functionalities of the software have now been established, we are now implementing core functionalities like the query store. We are also exploring how to establish a living open-source project that can bring the software to the status of a production release.

\section{Cross-University RDM Training}

Providing researchers and support staff with high-quality RDM training is essential to equip them with the skills and expertise demanded by data-intensive research work. To this end, FAIR Data Austria also develops crossuniversity training modules that are accessible through various media formats and are furthermore widely available and (re)usable in the form of Open Educational Resources (OER).

To streamline efforts and achieve results quickly, the project coordinators formed in December 2020 a dedicated Task Force which includes representatives from all partner universities. The Training Task Force developed a concept for cross-university training with three major components: (1) webinars on various aspects of RDM as part of the event series "Research Data Management in Austria", (2) a curated collection of OER on data management and digital preservation, (3) new or updated needsbased RDM courses at the individual universities.

Since June 2020, the project partners have held eight open online workshops with a total of over 600 participants. The target groups ranged from research support staff and service providers to researchers from specific disciplines. Both a privacy policy and consent forms for the instructors have been developed. Video recordings and presentation slides have been pu- 
blished as OER in Phaidra ${ }^{14}$ (the institutional repository of the University of Vienna) as well as on YouTube ${ }^{15}$. All materials are licensed under the CC-BY 4.0 International license so that they can be freely (re)used and adapted by other institutions and individuals alike willing to teach (and learn) more about RDM. They can also be incorporated into institutional training as part of e-learning, blended learning and flipped classroom programs.

The Training Task Force has also prepared multimedia information websites on RDM core topics, designed for researchers so that they can quickly familiarize themselves with the main aspects of good RDM practices. The curated collection of OER materials includes short videos with transcripts and documentation to ensure accessibility, as well as checklists, guides, interactive quizzes, and links to further information on each topic. The development of the OER materials was supported by interns from the University of Vienna Library, which took part of the new virtual internship program developed as part of the FAIR Data project (Kalová, 2021).

In April 2021, the three main partner universities (TU Graz, TU Wien, University of Vienna) joined the internationally renowned Carpentries community ${ }^{16}$ as "The Carpentries Austria" consortium. Nine project members have completed part one of the Carpentries instructor training ${ }^{17}$ so far, three of which are now certified Carpentries instructors. The aim of the Carpentries is to teach fundamental coding and data science skills to researchers and librarians around the globe.

Besides the Carpentries, FAIR Data Austria collaborates with other national and international initiatives on training, such as the OER Advanced $^{18}$ project, DINI/nestor UAG Schulungen ${ }^{19}$ and the FOSTER Open Science $^{20}$ project. Plans to develop an exciting new training project, to be announced later this year, are currently underway together with some of these initiatives.

Our experiences with the implementation of cross-university RDM training are outlined below:

- There is a growing interest in RDM training, as demonstrated by the comparatively large number of participants at our workshops (on average 75/webinar). In order to keep up with the raising demand for RDM training after the end of the project, more dedicated resources have to be provided at the institutional and national level.

- It is crucial to raise awareness about the ways and conditions to share sensitive data and/or metadata. Training in this area for researchers and support staff alike would be beneficial.

- Anyone developing virtual training should not underestimate the technical issues that might arise, including the recording and editing 
of videos. Testing all the tools before running a webinar unfortunately does not always guarantee everything will go smoothly on the day of the event. We therefore strongly advise using back-up recording software such as $\mathrm{OBS}^{21}$ when running online workshops.

- There is a plethora of high-quality open access materials on RDM available online, such as educasts on RDM topics. However, they are usually not easy to find, and they often lack the metadata, documentation, and license information necessary for reuse.

- The increase in virtual training caused by the pandemic has reinforced the view that interest in RDM training goes beyond country borders. Future training initiatives should therefore be developed through stronger international cooperation.

The Training Task Force has planned eight further virtual workshops ${ }^{22}$ for the winter semester of 2021/2022. The webinars will cover the following topics: Data Clearing House, FAIR Research Data, Repositories for Research Data (InvenioRDM), Data Management Plans in Technical Disciplines, Git, Open Educational Resources, FAIRification of Services, and Metadata for Research Data. Registration for the workshops will open in September 2021. The goal for the next few months is to invite other research institutions to offer webinars on additional RDM topics in the summer semester of 2022 to further establish the workshop series as a key source of introductory RDM training in Austria.

The OER collection will be published on the FAIR Office Austria ${ }^{23}$ website following a final review in the next months. The ongoing updates of existing RDM courses, together with design of new ones carried out by most of the project partners will be documented and made widely available as OER in 2022. This will contribute to an increased visibility of Austrian RDM training activities worldwide.

\section{Professionalization of Data Stewards at Austrian Universities}

Universities and other research institutions are fully aware that research is becoming increasingly data-intensive, interdisciplinary, and collaborative, and are therefore interested in retaining the value inherent in the vast amounts of research data. The new tasks and challenges brought by this interest has led to the establishment of Data Stewards, a new type of staff in academia who play the leading role in the adoption of RDM practices and expertise in an organisation. Data Stewards combine RDM knowledge 
with expertise in a specific research discipline. The approach to implement data stewardship programmes depends on the development status at an institution, its aims, and organizational structures. Our goal in FAIR Data Austria is to align data stewardship efforts and activities on a national and international level as well as define data steward models, profiles, competences, and training for the Austrian context.

Within the framework of the project, landscape studies, surveys and workshops have been conducted and organized by TU Graz to establish a concept for data stewardship programmes.

A survey has been conducted with all partner universities to collect input on current history, expectations and plans regarding data stewardship, to identify a possible route towards FAIR data management (Reichmann, 2020). An inter-university workshop between TU Wien, the University of Vienna and the Medical University Graz took place in October 2020 at TU Graz with the aim of defining and specifying Data Steward models for Austrian universities, together with corresponding profiles and tasks (Reichmann and Hasani-Mavriqi, 2021). In a follow-up workshop in April 2021, competences for data stewards were defined and mapped to predefined data steward models. Data Stewards of TU Graz provided insights into their current work and explained which competencies are needed, based on their experience (Gruber et.al., 2021). The latest workshop in July 2021 focused on suitable training opportunities where data stewards can acquire the competences needed. The following trainings were discussed and evaluated:

- Zertifikatskurs Forschungsdatenmanagement, TH Köln ${ }^{24}$

- Masterstudium Digitales Datenmanagement, Fachhochschule Potsdam und Humboldt University of Berlin ${ }^{25}$

- Postgraduate Certificate (Zertifikatskurs) Data Steward, University of Vienna ${ }^{26}$

- Data Train: Training in Research Data Management \& Data Science, U Bremen Research Alliance ${ }^{27}$

The TU Graz has employed three Data Stewards, with discipline-specific backgrounds in mechanical engineering, physics, and life sciences. They combine basic technical know-how and communication skills to interact successfully with both infrastructure experts and researchers, obtaining useful information from the former to provide the best support for the latter. Among their duties, which comprise organizational tasks, operational activities, and awareness-raising actions, the development of facultyspecific RDM policies is the most important, with the implementation of 
the first of its kind in Austria by the Faculty of Mechanical Engineering and Economics of TU Graz as one of its first major achievements ${ }^{28}$. The first measures of the new policy will be implemented by the end of 2021 . It is hoped that the policy developed can be used as a blueprint for other faculties and institutions.

In the fall of 2020, a "Data Stewardship Working Group", coordinated by the department Repository Management PHAIDRA-Services of the Vienna University Library, was established at the University of Vienna on behalf of the Vice-Rectorate for Digitization and Knowledge Transfer. This initiative included an analysis of vacancy listings for data stewards at various research institutions (Kalová and Gänsdorfer, 2021), as well as interviews with data stewards in Austria and other European countries (Gänsdorfer, 2020). The aim of both initiatives was to determine the most common tasks of data stewards, as well as the skills and competences necessary to complete them. Based on the results, the Working Group has developed a concept for institutional data stewardship at the University of Vienna. Furthermore, the University of Vienna Library is currently designing the first postgraduate certificate course for future data stewards, which will be open to researchers from all disciplines as well as research support staff. A demand-based curriculum is currently being designed to adequately prepare future data stewards for their tasks.

Our experience with the professionalization of Data Stewards at Austrian Universities shows that academic institutions have different prerequisites and requirements for the implementation of data stewardship programmes. Therefore, different models must be considered that take into account the different ways in which establishing data steward positions is handled by the universities, by e.g. repurposing existing positions or funding new ones.

After their implementation, Data Stewards face different challenges and expectations. Communication and awareness-raising for good RDM, together with clearly defined roles and responsibilities, remain challenges for all partners. Additionally, there is a high demand for data science and programming skills and competences.

The experience gained and the results obtained should be used to revise Data Steward training opportunities and to work further to match the requirements (university size, available resources, subject areas, number of DMPs/year, etc.) and existing solutions (number of Data Stewards, central/decentralised models, profiles, training...). The long-term goal is to establish a self-assessment toolkit that research institutions can use to identify and implement the data steward model that best suits them. 


\section{FAIR Office Austria}

The FAIR Office Austria initiative emerged from the FAIR Data Austria project has set itself the goal to connect stakeholders from research communities and service providers to jointly advance the implementation of the FAIR principles in Austria. More information can be found on https:// fair-office.at/ or via email at contact@fair-office.at.

The FAIR Office Austria website serves as information hub and facilitator of the FAIR principles in different scientific disciplines. To reflect the transdisciplinary and trans-institutional character of this initiative, the Office is managed and operated by three universities: TU Graz, TU Wien, University of Vienna in the core team. The Office is also conceived as a transnational interface that makes national activities that contribute to international efforts (EOSC ${ }^{29}$, GO FAIR ${ }^{30}, \mathrm{RDA}^{31}$ ) visible.

The launch event of FAIR Office Austria took place on $9^{\text {th }}$ June 2021. At the event, speakers from the GO FAIR initiative, Austrian Ministries and the participating universities engaged in multi-perspective discussions on the FAIR principles, with presentation of European initiatives and legal frameworks relevant for FAIR as well as the technical side of FAIR. Case studies were used to explain how the FAIR principles facilitate the handling of sensitive data and the cooperation between different institutions. FAIR Office Austria will support the exchange on FAIR topics in the Austrian research landscape. The video recording and the slides of the event are available through the repository Phaidra ${ }^{32}$.

During the international GO FAIR ${ }^{33}$ Festival in June 2021, FAIR Office Austria was accepted as one of the emerging national GO FAIR Offices, along with the Netherlands and Denmark. The Office will thus be part of the global GO FAIR initiative and will support its governance. At the local level, it will advocate for active participation in GO FAIR Implementation Networks (INs). INs are working groups dedicated to defining and creating specific materials and tools as elements of an "Internet of FAIR Data and Services" (IFDS).

When starting the FAIR Office Austria, it was planned to build a network of local reference points, initially found mainly in local research support units. During the project, other stakeholders such as interested researchers have come into focus as well, which has led the Office to expand the network to include all interested persons.

Engagement with other Austrian initiatives such as RDA Austria and the EOSC Mandated Organization have taken place, as FAIR is important to all supporting good RDM practices. As the national GO FAIR Office has 
just been founded recently, support of the GO FAIR International Support \& Coordination Office (GFISCO) and coordination with other national/ regional GO FAIR offices will be the next step, as well as the facilitation of the involvement of Austrian stakeholders in GO FAIR Implementation Networks.

In general, it is expected that the implementation and further development of FAIR Office Austria, the support of FAIR knowledge-building, and sharing of good implementation practices will continue in the future. This will be facilitated by contacting relevant initiatives and bodies in Austria (research performing institutions, funding organizations, ministries, industry, etc.) and further engagement with other Austrian initiatives. The Office will start a quarterly FAIR newsletter in September 2021, to keep interested people up to date on international and national development and upcoming events ${ }^{34}$.

\section{Conclusion}

This article gives an overview of the FAIR Data Austria project and describes the efforts of Austrian universities to create open and trustworthy environments for accessing and managing research data. FAIR Data Austria pursues the strengthening of knowledge transfer between universities, industry, and society, and supports the sustainable implementation of the European Open Science Cloud (EOSC) with the adoption of FAIR principles, which mandate that research data be Findable, Accessible, Interoperable, and Reusable.

\section{Acknowledgments}

The authors are very grateful to Miguel Rey Mazon for proofreading the article and providing valuable comments and suggestions. We would also like to thank Andreas Ferus and Peter Schaffer for their many valuable inputs on this work. This work is supported by the BMBWF funded project FAIR Data Austria (Digital and Social Transformation call, 2020-2024). 


\section{FAIR Data Austria}

Running Time: January 2020 to December 2022

\section{Project Lead: TU Graz}

Partners: TU Wien, University of Vienna, Academy of Fine Arts Vienna, Medical University Graz, and University of Innsbruck

Funding: Austrian Federal Ministry of Education, Science and Research (BMBWF)

\section{Objectives:}

- Integrated research data management (RDM) aligned with discipline-specific and general needs of research groups

- Establishment and development of tools for efficient creation of data management plans (DMPs) (machine-actionable DMPs maDMPs)

- Establishment and development of repositories for research data, code, and databases

- Professionalization of data stewards to develop the skills and expertise required for RDM

- Development of training and support services for efficient RDM

- Establishment of FAIR Office Austria

- Strengthening RDM at Austrian universities by bundling activities and visibility 
Mag. ${ }^{a}$ Dr. ${ }^{\text {in }}$ Susanne Blumesberger, MSc ORCID iD: https://orcid.org/0000-0001-9018-623X University of Vienna, Library and Archives E-Mail: susanne.blumesberger@univie.ac.at

Mag. Nikos Gänsdorfer University of Vienna, Library and Archives

Dipl.-Ing. (FH) Raman Ganguly ORCID iD: https://orcid.org/0000-0002-9837-0047 University of Vienna, Vienna University Computer Center E-Mail: raman.ganguly@univie.ac.at

Eva Gergely, BSc

ORCID iD: https://orcid.org/0000-0003-4218-5990 University of Vienna, Vienna University Computer Center E-Mail: eva.gergely@univie.ac.at

Dipl.-Ing. Alexander Gruber, BSc ORCID iD: https://orcid.org/0000-0002-4244-3446 Graz University of Technology, Institute of Interactive Systems and Data Science E-Mail: alexander.gruber@tugraz.at

Dipl.-Ing. in Dr. ${ }^{\text {in }}$ Ilire Hasani-Mavriqi ORCID iD: https://orcid.org/0000-0003-0758-0805 Graz University of Technology, Institute of Interactive Systems and Data Science E-Mail: ilire.hasani-mavriqi@tugraz.at

Tereza Kalová, MA (Res), M.A. LIS ORCID iD: https://orcid.org/0000-0002-1764-7228 University of Vienna, Library and Archives

E-Mail: tereza.kalova@univie.ac.at

Christopf Ladurner, BSc ORCID iD: https://orcid.org/0000-0003-3653-7558 Graz University of Technology, Library and Archives E-Mail: christoph.ladurner@tugraz.at 
Therese Macher, BSc MSc

Medical University of Graz,

Research Documentation and Research Evaluation

E-Mail: therese.macher@medunigraz.at

Tomasz Miksa, PhD

ORCID iD: https://orcid.org/0000-0002-4929-7875

TU Wien, Institute of Software Technology and Interactive Systems

E-Mail: tomasz.miksa@tuwien.ac.at

Mag. ${ }^{a}$ Barbara Sánchez Solis

ORCID iD: https://orcid.org/0000-0002-3574-2755

TU Wien, Center for Research Data Management

E-Mail: barbara.sanchez@tuwien.ac.at

Dipl.-Ing. Dr. Hermann Schranzhofer ORCID iD: https://orcid.org/0000-0003-0249-2726

Graz University of Technology, Institute of Interactive Systems and Data Science E-Mail: hermann.schranzhofer@tugraz.at

Dipl.-Ing. ${ }^{\text {in }}$ Christiane Stork ORCID iD: https://orcid.org/0000-0003-3176-4305 TU Wien, Center for Research Data Management E-Mail: christiane.stork@tuwien.ac.at

Sarah Stryeck, BSc MSc PhD ORCID iD: https://orcid.org/0000-0001-7524-6038

Graz University of Technology, Institute of Interactive Systems and Data Science

E-Mail: sarah.stryeck@tugraz.at

Dipl.-Soz. ${ }^{\text {in }}$ Heike Thöricht ORCID iD: https://orcid.org/0000-0002-1822-7559 University of Innsbruck, Information Technology Services (IT-Center) E-Mail: heike.thoericht@uibk.ac.at 


\section{Literature}

Gänsdorfer, N. (2020). Gespräche mit Data Stewards: Anforderungen, Kompetenzen, Aufgaben. https://phaidra.univie.ac.at/o:1137743

Gruber, A., Schranzhofer, H., Knopper, S., Stryeck, S. und Hasani-Mavriqi, I. (2021). Kompetenzen von Data Stewards an österreichischen Universitäten. Mitteilungen der Vereinigung Österreichischer Bibliothekarinnen und Bibliothekare, 74(1), 12-32. https://doi.org/10.31263/ voebm.v74i1.6255

Kalová, T. and Gänsdorfer, N. (2021). Die Rolle von Data Stewards: Analyse aktueller Stellenausschreibungen. https://doi.org/10.25365/ phaidra. 286

Kalová, T. (2021). Bibliothekspraktikum während der COVID-19-Pandemie: Erfahrungen mit Online-Praktika an der Universitätsbibliothek Wien. Mitteilungen der Vereinigung Österreichischer Bibliothekarinnen und Bibliothekare, 73(3-4), 422-434. https://doi.org/10.31263/voebm.v73i3-4.5371

Miksa, T., Simms, S., Mietchen, D., and Jones, S. (2019). Ten principles for machine-actionable data management plans. PLOS Computational Biology, 15(3), e1006750. https://doi.org/10.1371/journal. pcbi. 1006750

Miksa, T., Walk, P., Neish, P. (2020). RDA DMP Common Standard for Machine-actionable Data Management Plans. http://doi.org/10.15497/ rda00039

Rauber, A.; Asmi, A.; van Uytvanck, D., Pröll, S. (2015). Data Citation of Evolving Data: Recommendations of the Working Group on Data Citation (WGDC). https://doi.org/10.15497/RDA00016

Reichmann, S. (2020). Data Stewardship Profile - Results from a survey of 6 Austrian research-performing institutions. https://doi.org/10.25365/ phaidra. 242

Reichmann, S., Hasani-Mavriqi, I. (2021). Entwicklung eines Konzepts für Data Stewards an österreichischen Universitäten. https://doi. org/10.25365/phaidra.243 
* Contributors are named alphabetically; corresponding author: Ilire Hasani-Mavriqi.

1 FAIR Data Austria: https://forschungsdaten.at/en/fair-data-austria/

2 Zeno Casellato, DAMAP Tool: https://www.rd-alliance.org/system/ files/documents/1-Zeno-FairDataAustria-DMAP.pdf

3 re3data.org - Registry of Research Data Repositories: https://www.re3data.org

4 Science Europe, Practical Guide to the International Alignment of Research Data Management - Extended Edition: https://www.scienceeurope.org/our-resources/practical-guide-to-the-international-alignment-of-research-data-management/

5 euroCRIS, CERIF in Brief: https://www.eurocris.org/eurocris_archive/ cerifsupport.org/cerif-in-brief/index.html

6 National Center for Data to Health (CD2H), InvenioRDM: https:// cd2h.org/node/158

7 InvenioRDM: https://inveniordm.docs.cern.ch/

8 InvenioRDM, Features: https://inveniordm.docs.cern.ch/features/

9 TU Graz Repository: https://repository.tugraz.at/

10TU Graz Library: https://www.tugraz.at/tu-graz/universitaet/bibliothek-der-tu-graz/bibliothek-der-tu-graz/

11 Research Data Management at TU Graz: https://www.tugraz.at/sites/ rdm/home/

12 TU Wien Research Data: https://researchdata.tuwien.ac.at

13 Bauer-Marschallinger, B., Cao, S., Navacchi, C., Freeman, V., Reuß, F., Geudtner, D., Rommen, B., Vega, F. C., Snoeij, P., Attema, E., Reimer, C., \& Wagner, W. (2021). The Sentinel-1 Global Backscatter Model (S1GBM) - Mapping Earth's Land Surface with C-Band Microwaves (1.0) [Data set]. TU Wien. https://doi.org/10.48436/n2d1v-gqb91

14 RDM Austria, Veranstaltungsreihe „Forschungsdatenmanagement in Österreich": https://phaidra.univie.ac.at/view/o:1168881

15 FAIR Data Austria on YouTube: https://www.youtube.com/channel/ UC6UGkAxSQDhL8fQ0WT48Icw/videos

16 The Carpentries: https://carpentries.org/

17 The Carpentries, Teaching with The Carpentries: https://carpentries. org/teach/

18 Open Education Austria: https://www.openeducation.at/

19 Forschungsdaten.org, UAG Schulungen/Fortbildungen: https://www. forschungsdaten.org/index.php/UAG_Schulungen/Fortbildungen

20 FOSTER: https://www.fosteropenscience.eu/

21 OBS Studio: https://obsproject.com/de 
22 FAIR Data Austria, Materials: https://forschungsdaten.at/en/fair-data-austria/materials/

23 FAIR Office Austria, Learn More About FAIR: https://fair-office.at/index.php/information-for-researchers/learn-more-about-fair/?lang=en

24 TH Köln, Zertifikatskurs Forschungsdatenmanagement: https://www. th-koeln.de/weiterbildung/zertifikatskurs-forschungsdatenmanagement_82048.php

25 FH Potsdam, Digitales Datenmanagement, Studieninhalte: https:// www.ddm-master.de/studieninhalte/

26 As the course is currently being developed, there are no further information available at this time.

27 Universität Bremen, Data Train-Training in Research Data Management and Data Science: https://www.uni-bremen.de/research-alliance/forschungsdaten/data-train

28 TU Graz, RDM, Faculty Policies: https://www.tugraz.at/sites/rdm/policies/faculty-policies

29 European Open Science Cloud (EOSC): https://ec.europa.eu/info/research-and-innovation/strategy/goals-research-and-innovation-policy/ open-science/european-open-science-cloud-eosc_en

30 GO FAIR: https://www.go-fair.org/

31 Research Data Alliance (RDA): https://rd-alliance.org/

32 FAIR Data Austria, Video recording of FAIR Office Austria Launch Event: https://hdl.handle.net/11353/10.1201065

33 FAIR Festival 2021: https://www.go-fair.org/events/fair-festival-2021/

34 Sign up for the FAIR Office Austria newsletter at https://lists.univie. ac.at/mailman/listinfo/fairoffice_newsletter 